\title{
BMJ Open Acute subdural haematoma in the elderly: to operate or not to operate? A systematic review and meta-analysis of outcomes following surgery
}

\author{
Susruta Manivannan, ${ }^{1}$ Robert Spencer, ${ }^{2}$ Omar Marei, ${ }^{3}$ Isaac Mayo, ${ }^{4}$ Omar Elalfy, ${ }^{3}$ \\ John Martin, ${ }^{4}$ Malik Zaben (1) ${ }^{4}$
}

To cite: Manivannan S, Spencer R, Marei 0, et al. Acute subdural haematoma in the elderly: to operate or not to operate? A systematic review and meta-analysis of outcomes following surgery. BMJ Open 2021;11:e050786. doi:10.1136/ bmjopen-2021-050786

- Prepublication history and additional supplemental material for this paper are available online. To view these files, please visit the journal online (http://dx.doi.org/10.1136/ bmjopen-2021-050786).

SM and RS contributed equally.

Received 28 February 2021 Accepted 06 October 2021

Check for updates

(C) Author(s) (or their employer(s)) 2021. Re-use permitted under CC BY-NC. No commercial re-use. See rights and permissions. Published by BMJ.

${ }^{1}$ Department of Neurosurgery, Southampton University Hospitals NHS Trust, Southampton, UK

${ }^{2}$ Department of Neurosurgery, Queen Elizabeth University Hospital, Glasgow, UK ${ }^{3}$ School of Medicine, Cardiff University, Cardiff, UK ${ }^{4}$ Department of Neurosurgery, University Hospital of Wales, Cardiff, UK

Correspondence to

Dr Malik Zaben;

zabenm@cardiff.ac.uk

\section{ABSTRACT}

Objectives Acute subdural haematoma (ASDH) is a devastating pathology commonly found on CT brain scans of patients with traumatic brain injury. The role of surgical intervention in the elderly has been increasingly questioned due to its associated morbidity and mortality. Therefore, a systematic review and meta-analysis of the literature to quantify the mortality and functional outcomes associated with surgical management of ASDH in the elderly was performed. Design/setting A multidatabase literature search between January 1990 and May 2020, and meta-analysis of proportions was performed to quantify mortality and unfavourable outcome (Glasgow Outcome scale 1-3; death/ severe disability) rates.

Participants Studies reporting patients aged 60 years or older. Interventions Craniotomy, decompressive craniectomy, conservative management.

Outcome measures Mortality and functional outcomes (discharge, long-term follow-up (LTFU)).

Results 2572 articles were screened, yielding 21 studies for final inclusion and 15 for meta-analysis. Pooled estimates of mortality were $39.83 \%$ (95\% Cl $32.73 \%$ to $47.14 \% ; 10$ studies, $308 / 739$ patients, $I^{2}=73 \%$ ) at discharge and $49.30 \%$ (95\% Cl $42.01 \%$ to $56.61 \%$; 10 studies, $277 / 555$ patients, $\mathrm{I}^{2}=63 \%$ ) at LTFU. Mean duration of follow-up was 7.1 months (range 2-12 months). Pooled estimate of percentage of poor outcomes was $81.18 \%$ (95\% $\mathrm{Cl} 75.61 \%$ to $86.21 \% ; 6$ studies, $363 / 451$ patients, $\left.I^{2}=45 \%\right)$ at discharge, and $79.25 \%(95 \% \mathrm{Cl}$ $72.42 \%$ to $85.37 \%$; 8 studies, $402 / 511$ patients, $I^{2}=66 \%$ ) at LTFU. Mean duration of follow-up was 6.4 months (range 2-12 months). Potential risk factors for poor outcome included age, baseline functional status, preoperative neurological status and imaging parameters.

Conclusions Outcomes following surgical evacuation of ASDH in patients aged 60 years and above are poor. This constitutes the best level of evidence in the current literature that surgical intervention for ASDH in the elderly carries significant risks, which must be weighed against benefits.

PROSPERO registration number CRD42020189508.

\section{INTRODUCTION}

Traumatic brain injury (TBI) is among the leading causes of mortality and morbidity worldwide. ${ }^{12}$ We are currently experiencing an increasing contribution of traumatic injuries in the elderly population towards the
Strengths and limitations of this study

- Critical appraisal of studies reporting both mortality and functional outcomes following surgical evacuation of acute subdural haematoma in the elderly.

- Examination of risk factors for poor outcome in included studies.

- Heterogeneity arising from potential differences in indications for surgical intervention.

- Potential reporting bias and heterogeneity in metaanalysis from inclusion of smaller retrospective studies.

incidence of major intracranial injuries. ${ }^{3-5}$ Statistical data from the Centers for Disease Control and Prevention demonstrated a $17 \%$ increase in accidental falls-related TBI between 2008 and 2017 in the USA. ${ }^{6}$ Importantly, mortality rates secondary to falls-related TBI were disproportionately higher in adults aged 75 years and above, at 54.08 per 100000 person-year, compared with 8 per 100000 in those aged less than 75 years. With estimates of approximately 1.5 billion individuals aged above 65 years by 2050 , there is an urgent need for effective and pragmatic management of elderly patients with TBI. $^{7}$ Acute subdural haematoma (ASDH) is a potentially life-threatening form of TBI encountered by emergency services, which is characterised by extra-axial collection of acute blood between the dura and underlying brain parenchyma. The elderly are particularly vulnerable due to the risks of falls-related TBI, age-related atrophic changes in the brain and the use of anticoagulants. ${ }^{8}$ Hence, rationalisation of ASDH management in this group of patients is becoming increasingly pertinent. ${ }^{9} 10$ This was highlighted by a recent single centre retrospective study in the UK, which demonstrated that the odds of inpatient death were 
approximately 15 times higher than its chronic counterpart in patients aged 75 years and above. ${ }^{11}$

In the acute setting, ASDH management consists of either urgent surgical intervention or conservative management. The former typically consists of a craniotomy and evacuation of ASDH, though a decompressive craniectomy (DC) may be required in rare instances where the brain is severely swollen intraoperatively. However, the decision for surgical intervention for ASDH in elderly patients remains a point of contention due to its associated mortality. ${ }^{12}$ Surgical intervention is typically guided by imaging parameters including an ASDH $>10 \mathrm{~mm}$ thickness or midline shift (MLS) $>5 \mathrm{~mm}$ on CT imaging, neurological deterioration and/or intracranial pressure monitoring. ${ }^{13}$ While these guidelines are applicable to the general management of adult TBI, their applicability to the elderly population is less clear. Quantifying surgical morbidity and mortality is vital for tailoring decisions to this age group and managing the expectations of relatives who may not appreciate the associated risks. Therefore, in this study, we perform a systematic review and meta-analysis of the literature to quantify the mortality associated with surgical management of ASDH in the elderly, and identify potential risk factors for poor outcome in this group.

\section{METHODS}

This study was performed in accordance with the Preferred Reporting Items for Systematic Reviews and Meta-Analyses 2020 Statement. ${ }^{14}$

\section{Literature search}

A multidatabase (PubMed, Embase and Cochrane Reviews) literature search was performed between January 1990 and May 2020 by authors OM and OE (figure 1). Conflict of opinion was settled by senior author MZ. The following search terms were used in varying combinations: "subdural h(a)ematoma", "subdural h(a)emorrhage", "outcome*", "compar*", "morbid*", "mortality*", "complication*”, "reoccur*”, "recur*”, "surg*”, "operati*”, "non(-)surgical”, "burrhole”, "crani*”, "old*”, "frail*”, "geri*", and "elder*" (online supplemental data 1). Eligible articles were restricted to English language only,

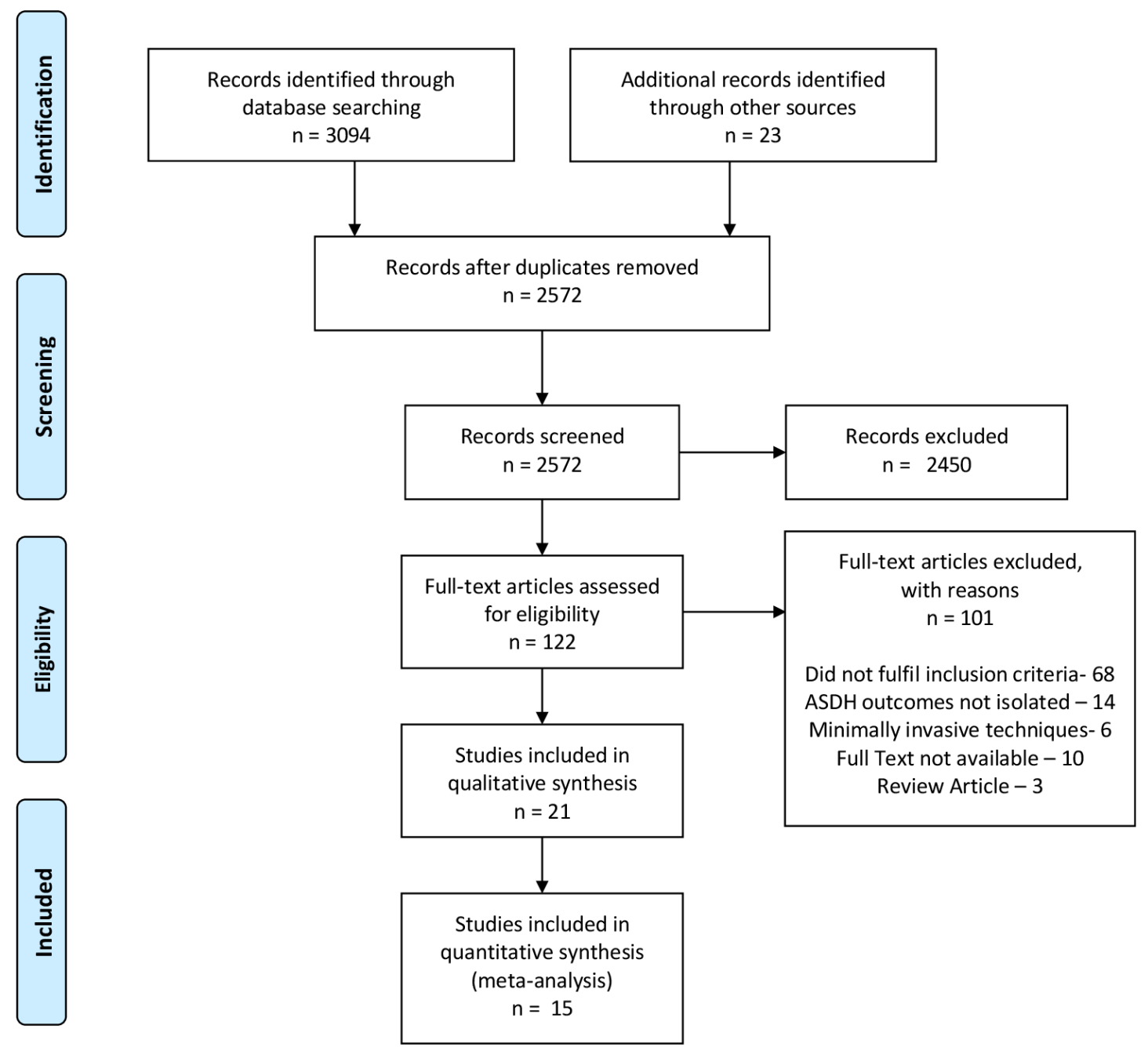

Figure 1 Search strategy for systematic literature review in accordance with PRISMA guidelines. ASDH, acute subdural haematoma; PRISMA, Preferred Reporting Items for Systematic Reviews and Meta-Analyses. 
and the bibliographies of included studies were screened for further relevant studies.

\section{Study selection}

Studies fulfilling the following criteria were included: (1) diagnosis of traumatic ASDH (confirmed on CT imaging), (2) patient age of 60 years or above, (3) patients who underwent surgical evacuation of ASDH and (4) reported clinical outcome. Studies including patients younger than 60 years were only included if management and clinical outcomes were specifically reported for the target age group. Exclusion criteria included: (1) diagnosis of chronic subdural haematoma with no evidence of acute component; (2) significant life-threatening extracranial injuries and (3) abstracts, conference presentations, editorials and expert opinions.

\section{Data extraction}

The following variables of interest were extracted by authors SM and RS from eligible studies: number of patients, age, gender, baseline functional status, preadmission residence, comorbidities, use of antithrombotic agents (antiplatelets or anticoagulants), mechanism of injury, preoperative Glasgow Coma Score (GCS)/ pupillary reaction to light, imaging findings, indications for surgical intervention, timing of surgery, intervention performed (craniotomy or decompressive craniectomy), mortality (at discharge and longer-term follow-up (LTFU)), clinical outcome (at discharge and LTFU) and duration of follow-up. Variables were summarised with respect to whether included studies examined associations with outcome. Studies reporting mortality and/ or Glasgow Outcome scale (GOS) were considered for inclusion in meta-analysis (see below). When reported, GOS was used to dichotomise outcomes into good (GOS 4-5) and poor (GOS 1-3) categories. Studies reporting conservative management along with surgical management were examined for potential comparison between different treatment groups (see below). Studies reporting minimally invasive techniques for ASDH evacuation were included for narrative synthesis. All included studies were assessed for quality using the Strengthening of Reporting of Observational Studies in Epidemiology (STROBE) criteria $^{15}$ and an adaptation of the risk of bias in nonrandomised studies of interventions (ROBINS-I) tool ${ }^{16}$ by two authors independently (IM and JM) (table 1). Studies with STROBE score $<13$ or ROBINS-I score of critical risk of bias were deemed ineligible for inclusion in meta-analysis.

\section{Statistical analysis}

Statistical analysis was performed using R V.3.6.0, and the metafor and meta packages. ${ }^{17}$ The percentage of patients under the following categories were pooled for metaanalysis: (1) deaths at discharge, (2) deaths at LTFU, (3) poor outcome at discharge and (4) poor outcome at LTFU. Poor outcome was defined as patients with GOS 1-3. Studies using other scoring systems were not included for meta-analysis to avoid introducing bias from definition of outcome. LTFU was defined as any time point beyond the time of discharge (specific duration of follow-up was recorded). All studies reporting mortality and/or GOS (specific score or dichotomised) in patients aged 60 years or above that underwent surgical evacuation of ASDH were included for meta-analysis. Surgical evacuation consisted of either craniotomy or decompressive craniectomy.

Heterogeneity was quantified with the following tests: DerSimonian and Laird estimator ${ }^{18}$ to estimate betweenstudy variance $\left(\tau^{2}\right.$, Cochran's $\mathrm{Q}$ test and the $\mathrm{I}^{2}$ statistic. Given that $\mathrm{I}^{2}>25 \%$ in all analyses and there was potential heterogeneity between studies with respect to specific age groups, a random effects model with double-arcsine transformation ${ }^{17} 19$ was used. Pooled estimates were reported with 95\% CIs. Studies published prior to January 2000 were excluded from meta-analysis to avoid distortion of summary effect sizes due to differences in healthcare technology as a function of time. Subgroup analysis was performed using the year of the study as a moderator (pre-January and post-January 2000) with a mixed effects model $^{17}$ (online supplemental data 2). Differences between subgroup summary estimates were significantly different. Therefore, older studies were excluded on both theoretical and statistical premises. Conservative and surgical groups were not compared due to fundamental differences in treatment indications and baseline cohort characteristics, which were acknowledged by relevant studies (online supplemental data 3,4).

\section{RESULTS}

A total of 2572 articles were screened, yielding 21 studies for final inclusion ${ }^{20-40}$ (table 1$)$. A set of fifteen studies were suitable for meta-analysis, with varying subsets used for each separate analysis depending on available outcome data. All studies were single centre retrospective studies, except for a recent retrospective multicentre study performed in Italy involving 213 patients from 5 centres. $^{38}$

\section{Mortality}

Mortality was generally defined as death by a specific time point, except for one study that also included death or discharge to a hospice. ${ }^{34}$ Mortality at discharge $(381 / 820$ patients) and LTFU (322/622 patients) was reported by 13 studies, of which 10 studies were included for metaanalysis (figure 2). Duration of follow-up was provided by all studies that reported mortality at LTFU, with an mean of 7.4 months (range 2-18 months). The pooled estimate of percentage of deaths was $39.83 \%$ (95\% CI $32.73 \%$ to $47.14 \%)$ at discharge, and $49.30 \%$ (95\% CI $42.01 \%$ to $56.61 \%)$ at LTFU. Mean duration of follow-up in studies included for meta-analysis was 7.1 months (range 2-12 months) (table 2).

\section{Functional outcomes}

Poor outcome was defined as patients with GOS 1-3. GOS on discharge (poor outcome in 437/532 patients) 


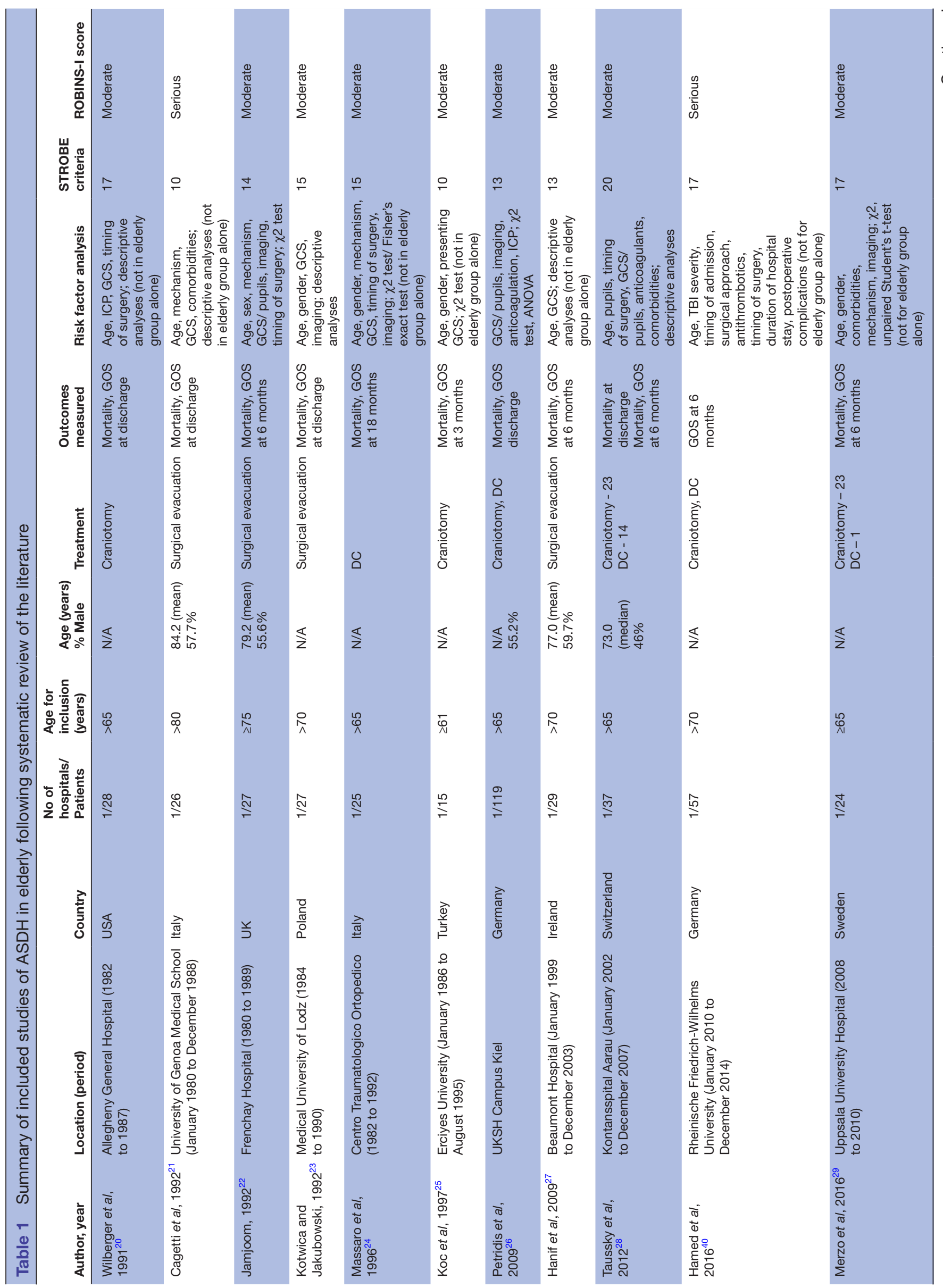




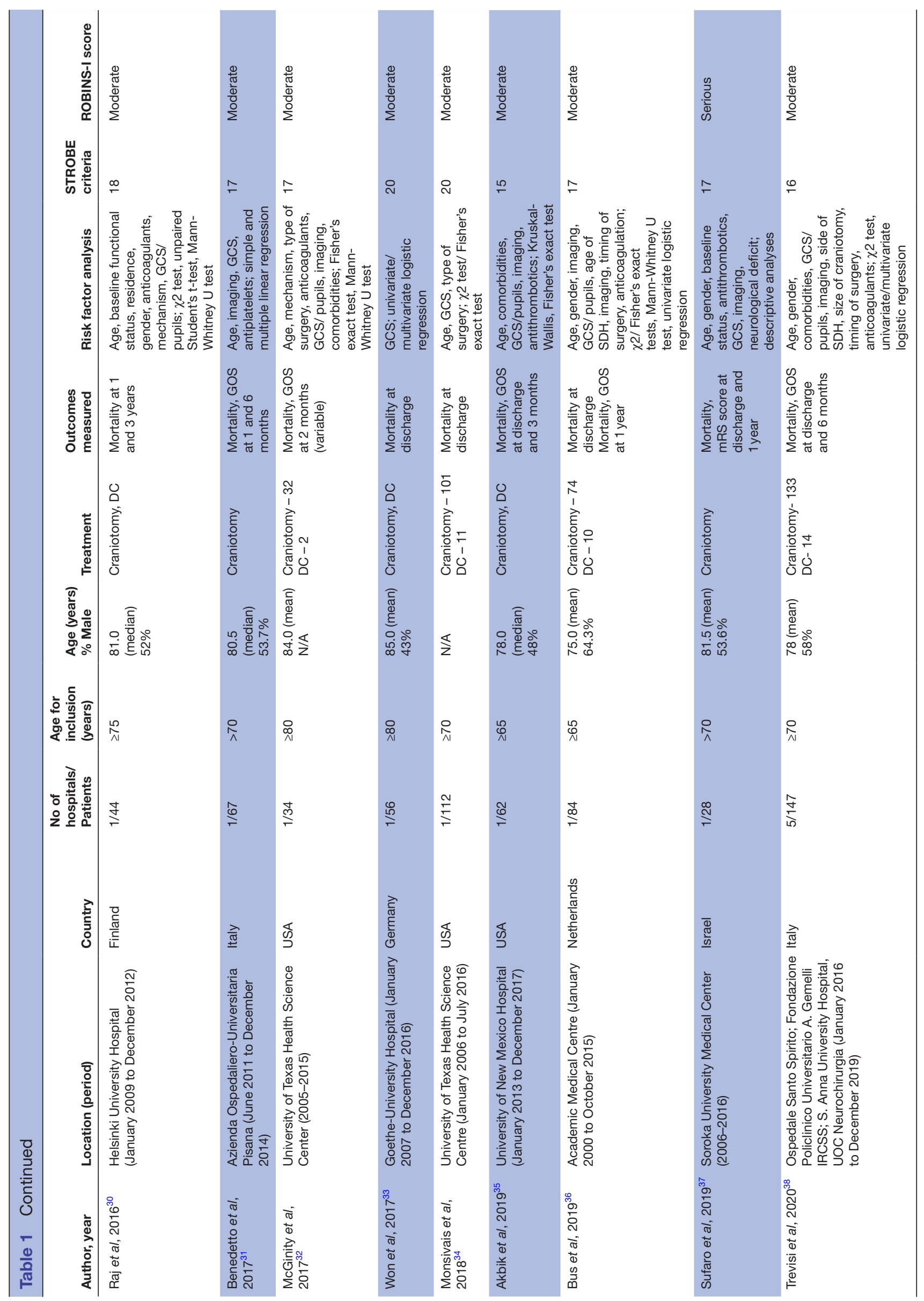




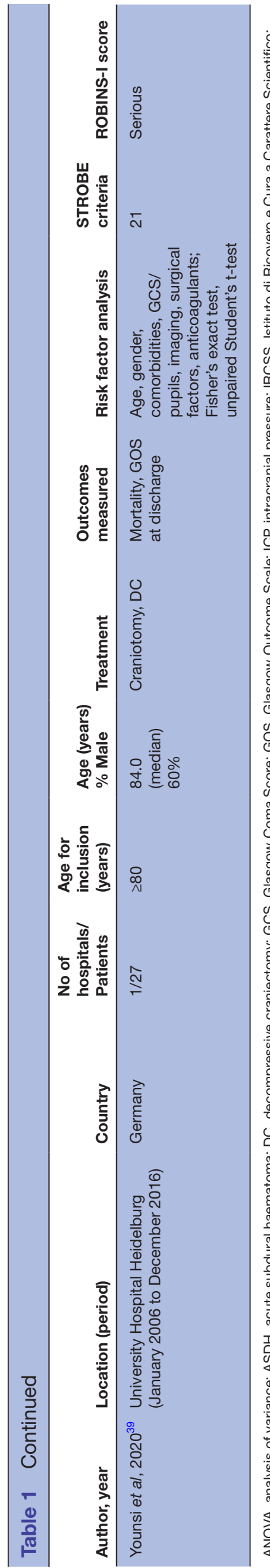

was reported by 9 studies, of which 6 were included for meta-analysis. GOS at LTFU (poor outcome in $459 / 578$ patients) was reported by 11 studies, of which 8 were included for meta-analysis (figure 2). Duration of follow-up was provided by all studies that reported GOS at LTFU, with a mean of 6.8 months (range 2-18 months). Pooled estimate of percentage of poor outcomes was $81.18 \%(95 \%$ CI $75.61 \%$ to $86.21 \%)$ at discharge, and $79.25 \%$ (95\% CI $72.42 \%$ to $85.37 \%$ ) at LTFU. Mean duration of follow-up in studies included for meta-analysis was 6.4 months (range 2-12 months) (table 2). One study ${ }^{37}$ reported modified Rankin Scale (mRS) scores at discharge and LTFU. Poor outcome was defined as mRS $5-6$, and reported in $28 \%$ (8/28 patients) at discharge and $57 \%(16 / 28$ patients $)$ at LTFU.

\section{Risk factors for poor outcome Demographics}

Inclusion age was reported by all studies, and analysed with respect to outcome in older age groups in nine studies (table 3 ). Of these, two studies reported a significant effect of age on outcome, ${ }^{31}{ }^{34}$ while the remainder reported no significant associations. With respect to GOS at 30 days, age was negatively associated with GOS on multivariate (regression coefficient $(\mathrm{RC})-0.054, \mathrm{p}=0.013$; other factors included volume of ASDH, GCS, MLS and antiplatelet use) but not univariate linear regression (RC $-0.043, p=0.095$ ) analyses. ${ }^{31}$ Age was negatively associated with GOS at 6 months on both univariate (RC -0.063 , $\mathrm{p}=0.031$ ) and multivariate ( $\mathrm{RC}-0.077, \mathrm{p}=0.002)$ linear regression analyses. ${ }^{31}$ Another study ${ }^{34}$ demonstrated a significant association between patient age group (70-79 years, 80 years and above) and mortality on univariate analysis $\left(p=0.05 ; \chi^{2}\right.$ test) and age $(70-79$ years vs 80 years and above) as a predictor of mortality on multivariate logistic regression (OR 2.83; 95\% CI 1.18 to 6.83 ; $\mathrm{p}=0.02$; other covariates included GCS and surgical approach). Gender was reported by 15 studies, and analysed with respect to outcome in 4 studies (table 3). A significant association with outcome was not demonstrated in any of these studies. ${ }^{30} 363839$

\section{Baseline functional status}

Baseline functional status was reported in 11 studies, of which 5 studies examined association with outcomes (table 3). One study ${ }^{39}$ included patients aged 80 years and above demonstrated a significant association between outcome (favourable, unfavourable) and number of comorbidities (more than 1, less than or equal to 1) ( $\mathrm{p}=0.05$; Fisher's exact test). This was not statistically significant on analysis with a greater number of comorbidities (more than or equal to 5, less than 5). In contrast, other studies reported no significant association between number of comorbidities and dichotomised GOS ${ }^{32} 38$ or mortality. ${ }^{34}$ Two studies demonstrated no statistically significant differences between survivors and non-survivors with respect to premorbid independence $^{3034}$ or preadmission residence. ${ }^{30}$ 


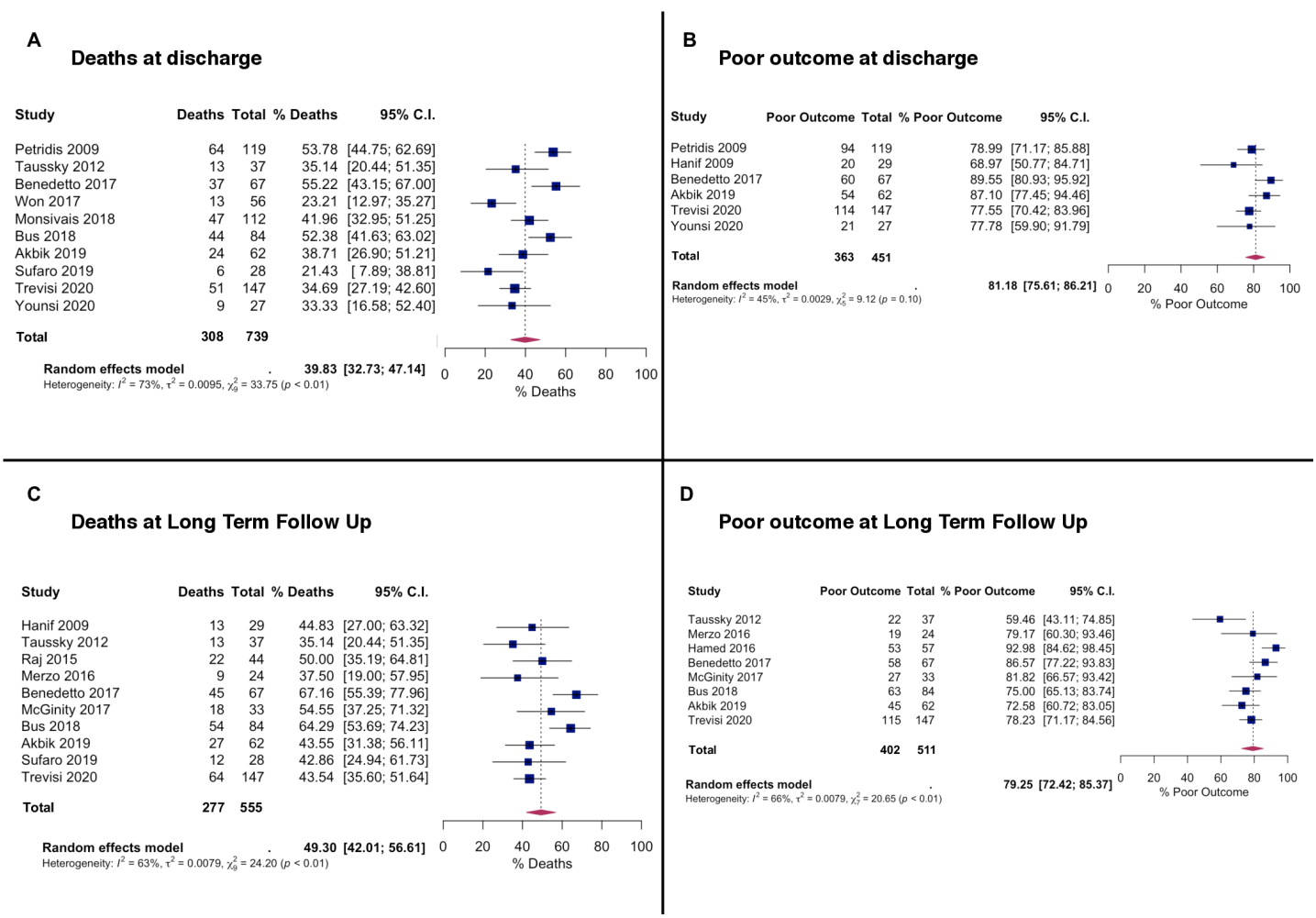

Figure 2 Forest plots depicting pooled estimates of mortality at discharge (A), poor outcome at discharge (B), mortality at discharge (C) and mortality at LTFU (D) in patients aged 65 years and above that underwent surgical evacuation of ASDH. ASDH, acute subdural haematoma; LTFU, long-term follow-up.

\section{Use of antithrombotic agents}

Use of antithrombotic agents was reported by 13 studies, of which 9 studies examined statistical association with outcome (table 3). Trevisi $e t a \hat{l}^{88}$ demonstrated a significant association between use of anti-thrombotic agents and dichotomised GOS on univariate analysis (82 vs $57 \%$; good vs poor outcome; $\mathrm{p}=0.01$ ), though multivariate logistic regression demonstrated no significant effect of antithrombotic use on dichotomised GOS (OR 0.33; 95\% CI 0.10 to 1.10 ; $\mathrm{p}=0.02$; other covariates included GCS, ASDH thickness and MLS). Other studies demonstrated no significant association between antithrombotic use and GOS, ${ }^{2635}$ dichotomised GOS ${ }^{32} 36$ or mortality. ${ }^{35}$ Examination of antiplatelet and anticoagulant use in isolation also demonstrated no significant association with GOS $^{3139}$ or mortality. ${ }^{34}$

\section{Mechanism and severity of injury}

Mechanism of injury was reported in seven studies. ${ }^{22}$ 26-28 303435 The association between mechanism of injury and outcome was examined in five of these studies, but a significant association was not demonstrated. ${ }^{22} 26283034$ In all seven studies reporting mechanism of injury, ground-level falls were the most common mechanism (range 65\%-100\%), with road traffic collisions being the second most common although much less common than falls (range 4\%-30\%).

Neurological status prior to surgical intervention was reported in all 21 included studies. Association between GCS or pupillary reactivity and outcome were examined in 11 studies. Of these, 10 studies demonstrated significant associations (table 3). Neurological status was reported preoperatively in two studies, ${ }^{22} 35$ on admission and preoperatively in two studies, ${ }^{36} 38$ and on admission in the remaining six studies. Significant heterogeneity was observed in the methodology used to assess the relationship between preoperative neurological status and outcome (online supplemental data 5,6). Statistically significant associations between GCS and GOS 3138 or mortality $^{26} 3033-35$ were demonstrated across multiple studies. Similarly, significant associations were also demonstrated between pupillary reactivity and GOS 2263536 or mortality. ${ }^{26}{ }^{35}$ No significant relationship between GCS and GOS was demonstrated in four studies, ${ }^{22} 323336$ and between pupil reactivity and GOS/ mortality in four studies. ${ }^{30} 323839$

Extracranial injuries were reported in four studies, ${ }^{21-23} 29$ while patients with significant extracranial injuries were excluded from five studies. ${ }^{2024253137}$ There were no reports of extracranial injuries in the remaining studies. None of the included studies examined the relationship between extracranial injuries and outcome. However, early studies demonstrated poor outcomes in several patients with concomitant injuries: (1) of six patients with orthopaedic fractures (three patients with femoral fractures, two patients with rib fractures and one patient with a C2 fracture), five patients died ${ }^{21}$ and (2) all eight patients with orthpaedic fractures had poor outcomes. ${ }^{22}$ 
Table 2 Mortality, outcomes and duration of follow-up following surgical evacuation of ASDH in patients aged 60 years and above

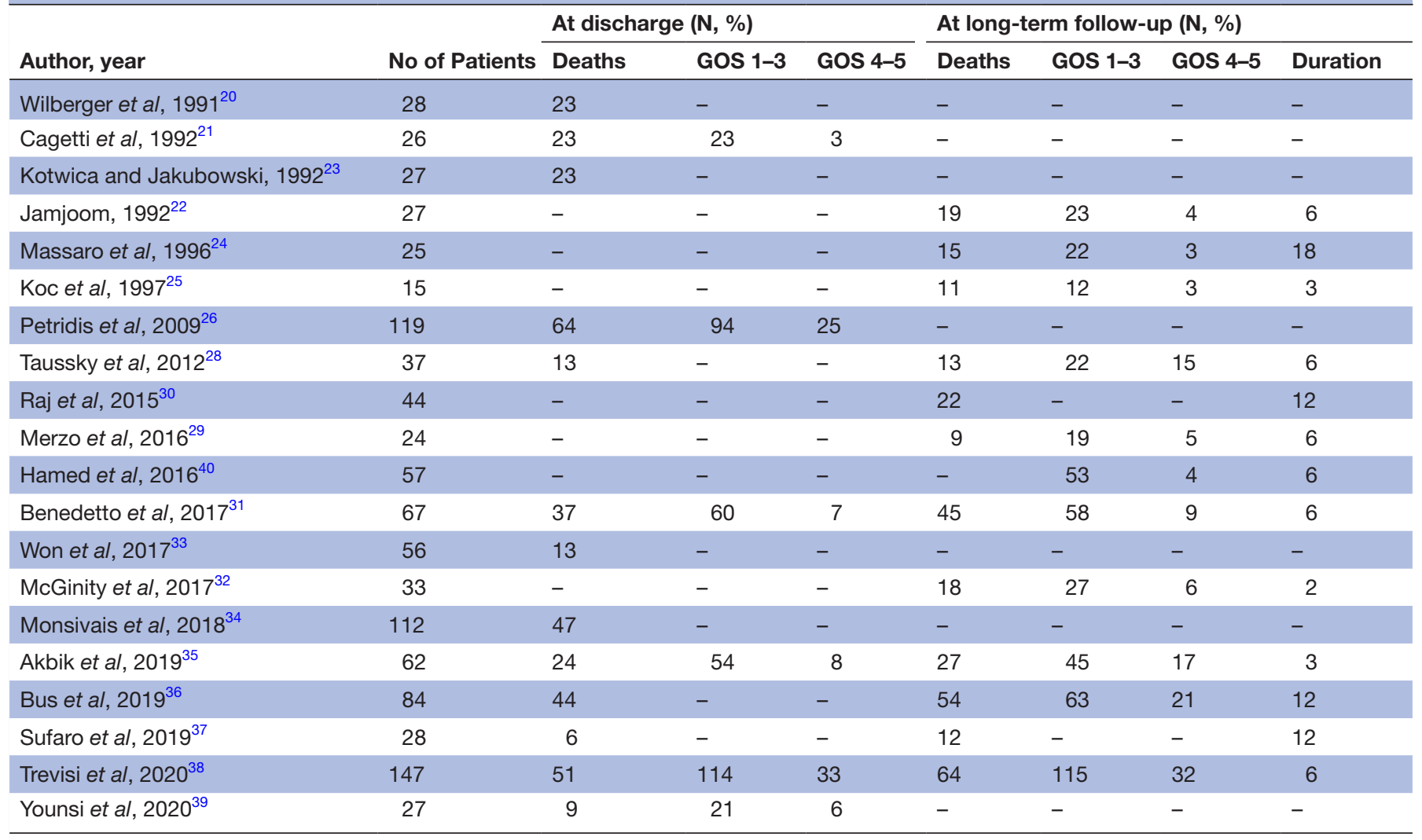

ASDH, acute subdural haematoma; GOS, Glasgow Outcome Scale.

\section{Imaging}

Imaging findings with respect to ASDH were reported in 15 included studies. The association between imaging findings and outcome was examined in eight of these studies, of which five showed significant findings (table 3 ). MLS was reported in seven studies, ${ }^{26} 313235363839$ while ASDH thickness or volume (estimated by volumetric analysis of CT scans) was reported in six studies. 263132363839 All studies reporting these imaging parameters analysed their association with outcome.

Petridis et $a l^{26}$ demonstrated a significantly greater proportion of patients with MLS $>10 \mathrm{~mm}$ in the GOS-1 than in the GOS-5 category $\left(\chi^{2}\right.$ test; $\left.\mathrm{p}<0.005\right)$. Furthermore, the difference between ASDH thickness and MLS was quantified and its association with outcome was examined. A significant association was identified between mean ASDH-MLS difference and individual GOS groups (analysis of variance (ANOVA); $\mathrm{p}<0.005$ ), measuring $6.3 \mathrm{~mm}$ in the GOS-5 and $1.8 \mathrm{~mm}$ in the GOS-1 category. Similarly, three other studies demonstrated a significant association between mean MLS and dichotomised GOS $^{3138}$ or mortality. ${ }^{35}$ One study demonstrated a negative association between MLS and GOS at both 30 days $(\mathrm{RC}-0.053, \mathrm{p}=0.015)$ and 6 months $(\mathrm{RC}-0.055, \mathrm{p}=0.026)$ on univariate linear regression, though this did not reach statistical significance on multivariate analysis. ${ }^{31}$ Two studies demonstrated no significant association between MLS and dichotomised ${ }^{39}$ or trichotomised ${ }^{35}$ GOS.

Two studies demonstrated significant associations between ASDH volume and outcome. ${ }^{31} 39$ One study demonstrated a negative association between ASDH volume and GOS at both 30 days $(\mathrm{RC}-0.006, \mathrm{p}=0.005)$ and 6 months $(\mathrm{RC}-0.005, \mathrm{p}=0.027)$ on univariate linear regression, though this did not reach statistical significance on multivariate analysis. ${ }^{31}$ Another study demonstrated a significantly smaller ASDH volume in the favourable outcome group when compared with the unfavourable outcome group $(67 \mathrm{~mL}$ vs $118 \mathrm{~mL}$; unpaired Student's t-test, $\mathrm{p}=0.05) .{ }^{39}$ Two studies demonstrated no significant association between ASDH thickness and GOS categories $^{26}$ or dichotomised GOS. ${ }^{38}$

The presence of additional intracranial injuries, such as cerebral contusions and traumatic subarachnoid haemorrhage, were reported in eight studies ${ }^{21-24} 2635$ 37-39 but only statistically analysed with respect to outcome in three studies. ${ }^{22} 2635$ None of these studies demonstrated significant associations between additional intracranial injuries and outcome.

\section{Surgical factors}

Timing of surgery from the time of admission or injury was reported in 10 studies, and analysed with respect to 


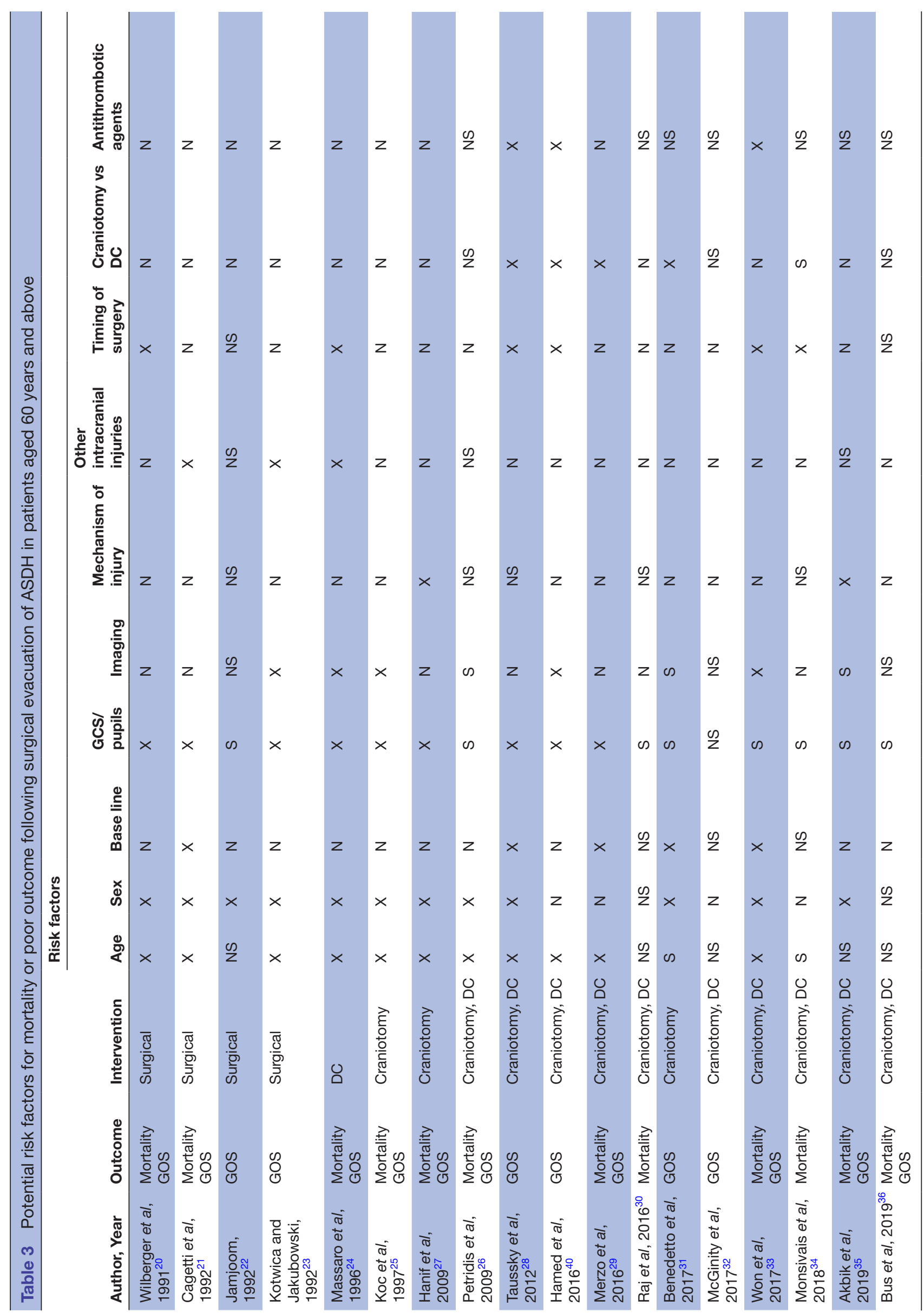




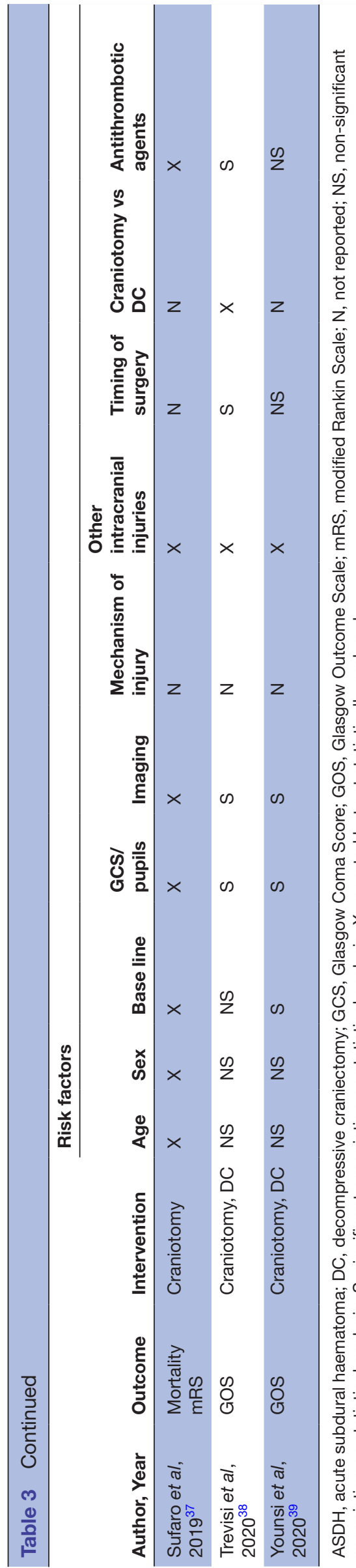

outcome in 4 studies (table 3 ). No statistically significant associations were demonstrated with respect to dichotomised GOS in three studies. ${ }^{223639}$ One study demonstrated a significant association of timing of surgery after admission, divided into 6 -hour increments between $<6$ hours and $>72$ hours, and GOS $\left(\chi^{2}\right.$ test; $\left.p<0.001\right) .{ }^{38}$ However, on univariate logistic regression, timing of surgery (within 6 hours or $>6$ hours), demonstrated no significant effect on prediction of dichotomised GOS $(\mathrm{p}=0.20)$.

Choice of surgical approach included craniotomy or DC. Four studies did not distinguish between craniotomy and decompressive craniectomy, but defined surgical intervention as the surgical evacuation of ASDH. ${ }^{20-23}$ Of the remaining studies, 12 studies included craniotomy and DC, 4 studies included craniotomies alone and 1 study defined surgical intervention as craniectomies ${ }^{24}$ (table 1). Number of patients undergoing each surgical approach was specifically reported in six studies, however, only three studies examined association with outcome (table 3). In total, 52 patients underwent DC, but outcomes were only reported for 23 patients. ${ }^{32} 3436$ Two studies reported poor outcomes in in 2 out of 2 , and 8 out of 10 patients. ${ }^{32}{ }^{36}$ One study reported mortality in 9 out of 11 patients. ${ }^{34}$ Monsivais $e t a l^{34}$ demonstrated a significant association between surgical approach (craniotomy, DC) and mortality on univariate analysis (Fisher's exact test; $\mathrm{p}<0.01$ ), and DC as a predictor of mortality when compared with craniotomy on multivariate logistic regression (OR 5.72; 95\% CI 1.11 to 29.32; $\mathrm{p}=0.04$; other covariates included age and GCS). Other studies demonstrated no significant associations between choice of surgical approach and dichotomised GOS $^{32} 36$ or mortality. ${ }^{36}$

\section{DISCUSSION \\ Surgical outcomes}

ASDH is a devastating traumatic pathology, and particularly so in the elderly. Although the significant mortality and morbidity associated with surgical intervention has been recognised for decades, there are no clear guidelines for ASDH management in this age group. With a growing elderly population worldwide and a shift in healthcare policy towards prioritising quality of life, it is essential that neurosurgical guidelines are adapted accordingly. ${ }^{9}{ }^{10}$ Indeed, the generic guidance for surgical intervention based on ASDH thickness, MLS and neurological deterioration may not necessarily be appropriate for elderly patients with ASDH. ${ }^{13}$ In this study, we systematically reviewed the literature for studies reporting outcomes following surgical intervention in patients aged 60 years and above with ASDH. We demonstrate that the pooled estimated mortality rate in this group is $40 \%$ at the point of discharge, and $49 \%$ at LTFU (figure 2). Furthermore, the pooled estimated poor outcome (GOS $1-3)$ rate is $81 \%$ at discharge and $79 \%$ at LTFU. Analysis of separate age subgroups could not be performed due to insufficient reported data, and therefore inclusion age of above 60 years was used as a minimum criterion for 
consideration. Only one study included patients above 60 years of age,${ }^{25}$ which was excluded from meta-analysis due to its date of publication. Therefore, our pooled estimated mortality and unfavourable outcome rates are generated from studies including patients aged 65 years and above. One potential source of heterogeneity was the inclusion of both craniotomy and DC within meta-analysis, as the majority of included studies did not distinguish reliably between surgical approach. Despite 11 studies including craniotomy and DC, only 7 studies reported specific numbers for each approach, and outcome could not be consistently deduced. We decided to include both procedures within the meta-analysis because: (1) we aimed to provide a pooled estimate for surgical intervention for ASDH in the elderly and (2) the decision between craniotomy and DC is intraoperative, therefore, quantification of mortality and morbidity following either procedure remains pertinent. In addition, of the three studies examining effect of surgical approach on GOS or mortality, only one study demonstrated a significant association with mortality. ${ }^{34}$ However, this was likely due to the small proportion of patients that underwent DC. Another source of heterogeneity was the duration of follow-up. For the pooled estimate of mortality at LTFU, duration of follow-up was 6 months in five studies, 12 months in three studies, 3 months in one study and 2 months in one study. Similarly, for the pooled estimate of poor GOS at LTFU, duration of follow-up was 6 months in four studies, 12 months in two studies, 3 months in one study and 2 months in one study. Therefore, pooled mortality may have been underestimated given that a higher percentage of deaths would be expected at longer durations of follow-up. While the same concept may be true for pooled estimated poor outcomes, it is also possible that shorter durations of follow-up did not capture potential future neurological recovery. Given that included studies did not consistently report the direct cause of poor outcome or mortality, it remains difficult to extrapolate any meaningful conclusions regarding this factor. Surgical inclusion or exclusion criteria were detailed in 10 studies, and largely revolved around existing Brain Trauma Foundation Guidelines ${ }^{13}$ (online supplemental data 4 ). The possibility of comparing surgical and conservative was initially evaluated, as four studies reported outcomes in conservatively managed patients. ${ }^{28} 333738$ However, indications for conservative management were due to inoperable comorbidities, good neurological status, moribund clinical status or unspecified (online supplemental data $3)$. Therefore, surgical and conservative groups were not feasible for comparison.

\section{Risk factors}

We also examined risk factors for mortality and poor outcome in the target cohort. Of nine studies examining the relationship between age and outcome, only two studies demonstrated a statistically significant effect. ${ }^{31} 34$ One study demonstrated a negative association between age and GOS at 30 days and 6 months on multivariate regression. ${ }^{31}$ Whether GOS should be modelled as a continuous variable is arguable, however, as each increment does not represent an equal difference in functional status. Another study, however, demonstrated that the odds of death were almost three times higher in patients aged 80 years and above compared with patients aged 70-79 years. While age appears intuitively likely to be associated with poorer outcomes, few studies demonstrate a significant association in the elderly cohort. Only two studies examined the relationship between baseline functional status and outcome. Neither demonstrated any difference between survivors and non-survivors with respect to premorbid independence ${ }^{30} 34$ or preadmission residence. ${ }^{30}$ Further studies are required to elucidate this relationship, since the use of scoring systems such as Karnofsky Performance Status ${ }^{41}$ or Clinical Frailty Score ${ }^{42}$ may provide a feasible method to prognosticate surgical candidates. This has been demonstrated by a recent study, which highlighted the use of the Modified Frailty Index ${ }^{43}$ to predict 30 days mortality and 6 months unfavourable outcome. ${ }^{44}$ Similarly, four studies reported the relationship between comorbidities and outcome, though only one study used a formal scoring system for comorbidities. ${ }^{38}$ However, no significant association with dichotomised GOS was demonstrated. ${ }^{38}$ In a similar fashion to baseline functional status, further studies using scoring systems such as the Charlston Comorbidity Index ${ }^{45}$ would be useful for assessment for neurosurgical decision-making.

In contrast, the association between neurological status and outcome were examined in 11 studies. Although significant associations were demonstrated across several studies, a considerable degree of heterogeneity was observed in the approach to analysis (online supplemental data 5,6). GCS was modelled as a continuous variable, dichotomised at various points or categorised with respect to severity of TBI. However, the emerging theme was that a lower GCS on admission was associated with a greater risk of mortality. Some studies demonstrated a similar relationship with risk of poor GOS, ${ }^{31} 3839$ though this did not consistently reach statistically significance. ${ }^{22} 323336$ Therefore, identifying a cut-off for admission GCS could be an important avenue for future research to help identify patients that are highly unlikely to benefit from surgery. Pupillary reactivity to light was also examined across several studies. As expected, fixed and dilated pupils were often associated with poor outcomes and mortality (online supplemental data 6), although some studies revealed no significant association. This could be due to subjective errors on reporting of pupillary reactivity, which may be remedied in future studies with use of pupillometers. In the absence of a reliable cut-off for preoperative GCS, understanding the relationship between pupillary reactivity and outcome is essential for prognosticating intubated patients. Several studies examined the relationship between imaging findings and outcome, demonstrating a significant association between MLS and outcome in four studies. ${ }^{26313538}$ Similar 
to the assessment of preoperative neurology, approaches to analysis were varied, with no reliable conclusion with respect to a prognostic cut-off value for MLS. Interestingly, while ASDH thickness was not significantly associated with outcome, ${ }^{26}{ }^{38}$ ASDH volume was significantly associated with GOS in two studies. ${ }^{31} 39$ The reported negative association between ASDH-MLS differential and GOS may also warrant further investigation. ${ }^{26}$ This has been previously reported in a retrospective cohort study of ASDH patients, which demonstrated a $75 \%$ mortality rate in patients with an ASDH-MLS differential of $>5 \mathrm{~mm} .{ }^{46}$ In contrast, the group with an ASDH-MLS differential of $0 \mathrm{~mm}$ only had a $25 \%$ mortality rate. Therefore, further studies comparing different measurement approaches may yield useful information regarding prognostication.

\section{Limitations}

We acknowledge several limitations from our systematic review and meta-analysis. Although we performed a multidatabase literature search, 10 full-text articles could not be accessed, which may have reported valid data for inclusion. Furthermore, non-English studies were excluded, raising the potential for geographical bias. Despite assessment of risk factors across included studies, a subgroup analysis could not be performed due to significant variation in the reporting of relevant data. Future studies should ensure that this is addressed as developing risk stratification scores will be essential to identify suitable surgical candidates. Also, duration of follow-up varied between studies with a range of 2-18 months, therefore, pooled estimates of outcomes at LTFU do not give an accurate indication of timescale. The majority of included studies were deemed at moderate risk of bias, though two studies were at serious risk (online supplemental data 7). However, none of the included studies were at critical risk of bias, and were therefore eligible for inclusion in metaanalysis. Of note, two key studies ${ }^{478}$ could not be included due to failure to meet our eligibility criteria. Shibahashi et $a t^{47}$ compared outcomes following craniotomy and DC for ASDH across 1028 patients matched for age, gender, admission GCS and vital signs, and comorbidities. Interestingly, no differences in mortality between treatment groups in the elderly cohort were found. Indeed, this finding supports our approach to a pooled estimate for mortality on meta-analysis. This study was not included in our review due to it not presenting absolute mortality figures, rather a mean difference between groups only. While it provides some evidence of no difference in mortality when comparing craniotomy with DC, the groups were not matched for pupillary abnormalities, nor were any imaging parameters taken into account in the matching process. One recent study was published outside of the dates of our search strategy, examining a total of 2508 patients aged 65 years and older that underwent surgical evacuation of ASDH. ${ }^{48}$ Data were collected from the USA National Trauma Data Bank registry between 2016 and 2017, which spans approximately 765 centres. Interestingly, inpatient mortality was estimated at only
$30.5 \%$. Multivariate logistic regression revealed that age, preoperative GCS, traumatic subarachnoid haemorrhage and MLS $>5 \mathrm{~mm}$ were significant predictors of mortality. Although the risk factors are in keeping with findings from previous studies, the reported inpatient mortality was comparatively low. Given that surgical approach was not specified, as International Classification of Diseases (ICD)-10 diagnosis codes were used to extract data, and surgical indications may have varied significantly between centres, it is unclear whether inpatient mortality is truly representative of this population.

\section{CONCLUSION}

In this systematic review and meta-analysis, we demonstrate that outcomes following surgical evacuation of ASDH in patients aged 65 years and above are poor. Pooled estimated mortality rates in this group are $40 \%$ at discharge and $49 \%$ at LTFU. Estimated rates of poor GOS are $81 \%$ at discharge and $79 \%$ at LTFU. Potential risk factors for poor outcome include age, baseline functional status, preoperative neurological status and imaging parameters. While further studies are required to reliably characterise predictors of poor outcome to inform separate guidelines for surgical intervention in this cohort, this systematic review constitutes the best level of evidence in the current literature that surgical intervention for ASDH in the elderly carries significant risks, which must be weighed against benefits. We, herein, provide robust evidence that helps healthcare professional decision making and counselling of families on a contentious issue.

Acknowledgements $\mathrm{MZ}$ is a member of the BRAIN Unit, which is funded by the Welsh Government through Health and Care Research Wales.

Contributors SM: data curation, formal analysis, project administration, software, writing-original draft; RS: data curation, formal analysis, project administration, software, writing-original draft; 0M: investigation, methodology; IM: investigation methodology; OE: investigation, methodology; JM: investigation, methodology, writing-reviewing and editing; MZ: conceptualisation, supervision, writing-review and editing, guarantor.

Funding The authors have not declared a specific grant for this research from any funding agency in the public, commercial or not-for-profit sectors.

Competing interests None declared.

Patient consent for publication Not applicable.

Provenance and peer review Not commissioned; externally peer reviewed.

Data availability statement Data are available on reasonable request. Data are available on request to the corresponding author: MZ, Neuroscience and Mental Health Research Institute (NMHRI), School of Medicine, University Hospital of Wales, Heath Park, Cardiff, CF14 4XN, email: ZabenM@cardiff.ac.uk.

Supplemental material This content has been supplied by the author(s). It has not been vetted by BMJ Publishing Group Limited (BMJ) and may not have been peer-reviewed. Any opinions or recommendations discussed are solely those of the author(s) and are not endorsed by BMJ. BMJ disclaims all liability and responsibility arising from any reliance placed on the content. Where the content includes any translated material, BMJ does not warrant the accuracy and reliability of the translations (including but not limited to local regulations, clinical guidelines, terminology, drug names and drug dosages), and is not responsible for any error and/or omissions arising from translation and adaptation or otherwise.

Open access This is an open access article distributed in accordance with the Creative Commons Attribution Non Commercial (CC BY-NC 4.0) license, which 
permits others to distribute, remix, adapt, build upon this work non-commercially, and license their derivative works on different terms, provided the original work is properly cited, appropriate credit is given, any changes made indicated, and the use is non-commercial. See: http://creativecommons.org/licenses/by-nc/4.0/.

\section{ORCID iD}

Malik Zaben http://orcid.org/0000-0002-7446-4532

\section{REFERENCES}

1 Rubiano AM, Carney N, Chesnut R, et al. Global neurotrauma research challenges and opportunities. Nature 2015;527:S193-7.

2 Majdan M, Plancikova D, Brazinova A, et al. Epidemiology of traumatic brain injuries in Europe: a cross-sectional analysis. Lancet Public Health 2016;1:e76-83.

3 Roozenbeek B, Maas AIR, Menon DK. Changing patterns in the epidemiology of traumatic brain injury. Nat Rev Neurol 2013;9:231-6.

4 Beck B, Gantner D, Cameron PA, et al. Temporal trends in functional outcomes after severe traumatic brain injury: 2006-2015. J Neurotrauma 2018;35:1021-9.

5 Majdan M, Mauritz W. Unintentional fall-related mortality in the elderly: comparing patterns in two countries with different demographic structure. BMJ Open 2015;5:e008672.

6 Peterson AB, Kegler SR. Deaths from fall-related traumatic brain injury - United States, 2008-2017. MMWR Morb Mortal Wkly Rep 2020;69:225-30.

7 UNDoEaS. World population prospects 2019: highlights (ST/ ESA/SER.A4423), 2019. Available: https://population.un.org/wpp/ Publications/Files/WPP2019_Highlights.pdf [Accessed 02 Jan 2021].

8 Won S-Y, Dubinski D, Bruder M, et al. Acute subdural hematoma in patients on oral anticoagulant therapy: management and outcome. Neurosurg Focus 2017;43:E12.

9 Evans LR, Jones J, Lee HQ, et al. Prognosis of acute subdural hematoma in the elderly: a systematic review. J Neurotrauma 2019;36:517-22

10 Christensen K, Doblhammer G, Rau R, et al. Ageing populations: the challenges ahead. Lancet 2009;374:1196-208.

11 Whitehouse KJ, Jeyaretna DS, Enki DG, et al. Head injury in the elderly: what are the outcomes of neurosurgical care? World Neurosurg 2016;94:493-500.

12 Ushewokunze S, Nannapaneni R, Gregson BA, et al. Elderly patients with severe head injury in coma from the outset--has anything changed? Br J Neurosurg 2004;18:604-7.

13 Bullock MR, Chesnut R, Ghajar J, et al. Surgical management of acute subdural hematomas. Neurosurgery 2006;58:S2-16-S2-24.

14 Page MJ, McKenzie JE, Bossuyt PM, et al. The PRISMA 2020 statement: an updated guideline for reporting systematic reviews. BMJ 2021;372:n71.

15 von Elm E, Altman DG, Egger M, et al. The strengthening the reporting of observational studies in epidemiology (STROBE) statement: guidelines for reporting observational studies. J Clin Epidemiol 2008;61:344-9.

16 Sterne JA, Hernán MA, Reeves BC, et al. ROBINS-I: a tool for assessing risk of bias in non-randomised studies of interventions. BMJ 2016;355:i4919.

17 Wang N. Conducting meta-analyses of proportions in R. 2017, 2020. Available: www.wangnaike.com [Accessed 10 Jan 2020].

18 DerSimonian R, Laird N. Meta-Analysis in clinical trials. Control Clin Trials 1986;7:177-88.

19 Barendregt JJ, Doi SA, Lee YY, et al. Meta-Analysis of prevalence. J Epidemiol Community Health 2013:67:974-8.

20 Wilberger JE, Harris M, Diamond DL. Acute subdural hematoma: morbidity, mortality, and operative timing. J Neurosurg 1991;74:212-8.

21 Cagetti B, Cossu M, Pau A, et al. The outcome from acute subdural and epidural intracranial haematomas in very elderly patients. $\mathrm{Br} \mathrm{J}$ Neurosurg 1992;6:227-31.

22 Jamjoom A. Justification for evacuating acute subdural haematomas in patients above the age of 75 years. Injury 1992;23:518-20.

23 Kotwica Z, Jakubowski JK. Acute head injuries in the elderly. An analysis of 136 consecutive patients. Acta Neurochir 1992;118:98-102.

24 Massaro F, Lanotte M, Faccani G, et al. One hundred and twenty-seven cases of acute subdural haematoma operated on. Correlation between CT scan findings and outcome. Acta Neurochir 1996;138:185-91.
25 Koç RK, Akdemir H, Oktem IS, et al. Acute subdural hematoma: outcome and outcome prediction. Neurosurg Rev 1997;20:239-44.

26 Petridis AK, Dörner L, Doukas A, et al. Acute subdural hematoma in the elderly; clinical and CT factors influencing the surgical treatment decision. Cent Eur Neurosurg 2009;70:73-8.

27 Hanif S, Abodunde O, Ali Z, et al. Age related outcome in acute subdural haematoma following traumatic head injury. Ir Med $J$ 2009;102:255-7

28 Taussky P, Hidalgo ET, Landolt $\mathrm{H}$, et al. Age and salvageability: analysis of outcome of patients older than 65 years undergoing craniotomy for acute traumatic subdural hematoma. World Neurosurg 2012;78:306-11.

29 Merzo A, Lenell S, Nyholm L, et al. Promising clinical outcome of elderly with TBI after modern neurointensive care. Acta Neurochir 2016;158:125-33.

30 Raj R, Mikkonen ED, Kivisaari R, et al. Mortality in elderly patients operated for an acute subdural hematoma: a surgical case series. World Neurosurg 2016;88:592-7.

31 Benedetto N, Gambacciani C, Montemurro N, et al. Surgical management of acute subdural haematomas in elderly: report of a single center experience. Br J Neurosurg 2017;31:244-8.

32 McGinity MJ, Michalek JE, Rodriguez JS, et al. Surgical evacuation of acute subdural hematoma in octogenarians: a ten-year experience from a single trauma center. Br J Neurosurg 2017;31:714-7

33 Won S-Y, Dubinski D, Brawanski N, et al. Significant increase in acute subdural hematoma in octo- and nonagenarians: surgical treatment, functional outcome, and predictors in this patient cohort. Neurosurg Focus 2017;43:E10.

34 Monsivais D, Choi HA, Kitagawa R, et al. A retrospective analysis of surgical outcomes for acute subdural hematoma in an elderly cohort. Interdisciplinary Neurosurgery 2018;14:130-4.

35 Akbik OS, Starling RV, Gahramanov S, et al. Mortality and functional outcome in surgically evacuated acute subdural hematoma in elderly patients. World Neurosurg 2019;126:e1235-41.

36 Bus S, Verbaan D, Kerklaan BJ, et al. Do older patients with acute or subacute subdural hematoma benefit from surgery? Br J Neurosurg 2019;33:51-7.

37 Sufaro Y, Avraham E, Alguyn F, et al. Unfavorable functional outcome is expected for elderly patients suffering from acute subdural hematoma even when presenting with preserved level of consciousness. J Clin Neurosci 2019;67:167-71.

38 Trevisi G, Sturiale CL, Scerrati A, et al. Acute subdural hematoma in the elderly: outcome analysis in a retrospective multicentric series of 213 patients. Neurosurg Focus 2020;49:E21.

39 Younsi A, Fischer J, Habel C, et al. Mortality and functional outcome after surgical evacuation of traumatic acute subdural hematomas in octa- and nonagenarians. Eur J Trauma Emerg Surg 2021;47:14991510.

40 Hamed M, Schuss P, Daher FH, et al. Acute traumatic subdural hematoma: surgical management in the presence of cerebral Herniation-A single-center series and multivariate analysis. World Neurosurg 2016:94:501-6.

41 Mor V, Laliberte L, Morris JN, et al. The Karnofsky performance status scale. An examination of its reliability and validity in a research setting. Cancer 1984;53:2002-7.

42 Rockwood K, Song X, MacKnight C, et al. A global clinical measure of fitness and frailty in elderly people. CMAJ 2005;173:489-95.

43 Tsiouris A, Hammoud ZT, Velanovich V, et al. A modified frailty index to assess morbidity and mortality after lobectomy. J Surg Res 2013;183:40-6.

44 Lee H, Tan C, Tran V, et al. The utility of the modified frailty index in outcome prediction for elderly patients with acute traumatic subdura hematoma. J Neurotrauma 2020;37:2499-506.

45 Quan H, Li B, Couris CM, et al. Updating and validating the Charlson comorbidity index and score for risk adjustment in hospital discharge abstracts using data from 6 countries. Am J Epidemiol 2011;173:676-82.

46 Zumkeller M, Behrmann R, Heissler HE, et al. Computed tomographic criteria and survival rate for patients with acute subdural hematoma. Neurosurgery 1996;39:708-12.

47 Shibahashi K, Sugiyama K, Tomio J, et al. In-hospital mortality and length of hospital stay with craniotomy versus craniectomy for acute subdural hemorrhage: a multicenter, propensity score-matched analysis. J Neurosurg 2019:1-10.

48 Kerezoudis P, Goyal A, Puffer RC, et al. Morbidity and mortality in elderly patients undergoing evacuation of acute traumatic subdural hematoma. Neurosurg Focus 2020;49:E22. 